\title{
Nonlinear optimization of beam lines
}

\author{
R. Tomás \\ CERN, Geneva, Switzerland \\ (Received 20 April 2006; published 16 August 2006)
}

\begin{abstract}
The current final focus systems of linear colliders have been designed based on the local compensation scheme proposed by Raimondi and Seryi. However, there exist remaining aberrations that deteriorate the performance of the system. This paper develops a general algorithm for the optimization of beam lines based on the computation of the high orders of the transfer map using MAD-X and Polimorphic Tracking Code. The algorithm is applied to the CLIC beam delivery system.
\end{abstract}

DOI: 10.1103/PhysRevSTAB.9.081001

PACS numbers: 41.85.Gy, 29.27.Eg

\section{INTRODUCTION}

The minimization of aberrations in beam lines has always been a concern. Already in 1973 [1] an analytical approach was derived up to the second order but particle tracking had to be used for higher orders. More recently with the advent of the local compensation scheme [2] the demand for design and optimization algorithms that take into account higher orders has largely increased [3-6]. This paper describes a general optimization algorithm that takes as figure of merit the rms beam sizes at the end of the beam line. These are analytically computed from the coefficients of the transfer map to an arbitrary order. A particular application to the CLIC beam delivery system (BDS) [7] is shown as a proof of principle.

\section{MATHEMATICAL GROUND}

The transfer map between two locations of a beam line is expressed in the form

$$
x_{f}=\sum_{j k l m n} X_{j k l m n} x_{0}^{j} p_{x 0}^{k} y_{0}^{l} p_{y 0}^{m} \delta_{0}^{n},
$$

where $x_{f}$ represents any of the final coordinates $\left(x_{f}, p_{x f}\right.$, $\left.y_{f}, p_{y f}\right)$, the initial coordinates are represented with the zero subindex and $X_{j k l m}$ are the map coefficients of the corresponding final coordinate. The MAD-X [8] code together with the Polimorphic Tracking Code (PTC) [9] can provide $X_{j k l m}$ up to the desired order.

The quadratic standard deviation of the final density distribution $\left\langle x_{f}^{2}\right\rangle$ is given by the following integral:

$$
\left\langle x_{f}^{2}\right\rangle=\int x_{f}^{2} \rho_{f} d v_{f}
$$

where $d v_{f}$ represents the differential volume of the final phase space. Assuming that the transfer map is symplectic $\rho_{f} d v_{f}=\rho_{0} d v_{0}$ and using Eq. (1),

$$
\begin{aligned}
\left\langle x_{f}^{2}\right\rangle= & \sum_{\substack{j k k l m m \\
j^{\prime} l^{\prime} l^{\prime} m^{\prime} n^{\prime}}} X_{j k l m n} X_{j^{\prime} k^{\prime} l^{\prime} m^{\prime} n^{\prime}} \int x_{0}^{j+j^{\prime}} p_{x 0}^{k+k^{\prime}} y_{0}^{l+l^{\prime}} p_{y 0}^{m+m^{\prime}} \delta_{0}^{n+n^{\prime}} \\
& \times \rho_{0} d v_{0}
\end{aligned}
$$

To perform this integral a Gaussian centered bunch is assumed in the transverse planes and a rectangular distribution is considered in relative energy deviation (for the application to CLIC), given by the following expression:

$$
\rho_{0}=\frac{e^{-x^{2} / 2 \sigma_{x}^{2}} e^{-p_{x}^{2} / 2 \sigma_{p_{x}}^{2}} e^{-y^{2} / 2 \sigma_{y}^{2}} e^{-p_{y}^{2} / 2 \sigma_{p_{y}}^{2}} \Pi\left(\frac{\delta}{\Delta_{\delta}}\right)}{(2 \pi)^{2} \sigma_{x} \sigma_{p_{x}} \sigma_{y} \sigma_{p_{y}} \Delta_{\delta}},
$$

where $\Pi(z)$ is the rectangular function which vanishes when $|z|>0.5$ and takes the unit value elsewhere. This distribution is representative for the beam expected at the end of the CLIC linac. Note that assuming this particle density imposes constraints in the Twiss functions at the initial location. The horizontal and vertical alpha functions and the closed orbit must be zero. This assumption is fundamental to gain speed in the numerical computation of $\left\langle x_{f}^{2}\right\rangle$ as will be shown below. Moreover, this constraint is not critical since even in the case that the initial location has a nonzero $\alpha$ function it is possible to add a matching section meeting our constraints in the new initial point but leaving the initial beam line unchanged.

To compute the integral above the following results are used:

$$
\frac{1}{\sqrt{2 \pi} \sigma} \int z^{n} e^{-z^{2} / 2 \sigma^{2}} d z= \begin{cases}\Gamma\left(\frac{1+n}{2}\right) \sigma^{n} \sqrt{\frac{2^{n}}{\pi}} & \text { if } n \text { even } \\ 0 & \text { if } n \text { odd }\end{cases}
$$

$$
\frac{1}{\sigma} \int z^{n} \Pi\left(\frac{z}{\sigma}\right) d z= \begin{cases}\frac{1}{n+1}\left(\frac{\sigma}{2}\right)^{n} & \text { if } n \text { even, } \\ 0 & \text { if } n \text { odd, }\end{cases}
$$

where $\Gamma(z)$ is the Gamma function. From these equations the gain in computational speed thanks to the chosen symmetry is patent. Using the above equations the standard quadratic deviation of the particle distribution at the end of the beam line is given by 


$$
\begin{aligned}
\left\langle x_{f}^{2}\right\rangle= & \sum_{j k l m n} X_{j k l m n}^{2} \Gamma\left(\frac{1+2 j}{2}\right) \Gamma\left(\frac{1+2 k}{2}\right) \Gamma\left(\frac{1+2 l}{2}\right) \Gamma\left(\frac{1+2 m}{2}\right) \frac{2^{j+k+l+m-2 n}}{(2 n+1) \pi^{2}} \sigma_{x}^{2 j} \sigma_{p_{x}}^{2 k} \sigma_{y}^{2 l} \sigma_{p_{y}}^{2 m} \Delta_{\delta}^{2 n} \\
& +\sum_{j k l m n>j^{\prime} k^{\prime} l^{\prime} m^{\prime} n^{\prime}} 2 X_{j k l m n} X_{j^{\prime} k^{\prime} l^{\prime} m^{\prime} n^{\prime}} \Gamma\left(\frac{1+j+j^{\prime}}{2}\right) \Gamma\left(\frac{1+k+k^{\prime}}{2}\right) \Gamma\left(\frac{1+l+l^{\prime}}{2}\right) \Gamma\left(\frac{1+m+m^{\prime}}{2}\right) \\
& \times \frac{2^{\left[\left(j+k+l+m+j^{\prime}+k^{\prime}+l^{\prime}+m^{\prime}\right) / 2\right]-n-n^{\prime}}}{\left(n+n^{\prime}+1\right) \pi^{2}} \sigma_{x}^{j+j^{\prime}} \sigma_{p_{x}}^{k+k^{\prime}} \sigma_{y}^{l+l^{\prime}} \sigma_{p_{y}}^{m+m^{\prime}} \Delta_{\delta}^{n+n^{\prime}} .
\end{aligned}
$$

The following sections describe means to extract the information concerning the nature of the aberrations.

\section{A. The order-by-order approach}

By truncating the map at order $q$ we only consider the coefficients $X_{j k l m n}$ such that $j+k+l+m+n \leq q$. The resulting standard deviation is represented by $\left\langle x_{f}^{2}\right\rangle_{q}$. Thus defined, $\sqrt{\left\langle x_{f}^{2}\right\rangle_{1}}$ corresponds to rms size given by the linear Twiss functions, $\sqrt{\left\langle x_{f}^{2}\right\rangle_{2}}$ takes into account the effect of chromatic aberrations and sextupoles, $\sqrt{\left\langle x_{f}^{2}\right\rangle_{3}}$ incorporates octupolar fields, etc. The final finite size of the bunch is given by $\left\langle x_{f}^{2}\right\rangle_{q}$ when $q$ tends to infinite. However, there must be a finite order $p$ that gives a satisfactory approximation. The evaluation of $\left\langle x_{f}^{2}\right\rangle_{q}-\left\langle x_{f}^{2}\right\rangle_{q-1}$ gives the contribution of the order $q$ to the final rms beam size. From this contribution the order of the most relevant aberrations is inferred, and subsequently the appropriate multipolar correctors are chosen. However, the optimum location of the correctors still needs to be identified.

\section{B. Chromatic versus achromatic correctors}

This section gives the recipe to decide if the correctors should be placed in locations with or without dispersion. $\left\langle x_{f}^{2}\right\rangle_{q, \Delta_{\delta}=0}$ is defined as the rms size of a monochromatic beam; it is given by

$$
\begin{aligned}
\left\langle x_{f}^{2}\right\rangle_{q, \Delta_{\delta}=0}= & \sum_{\substack{j k l m m \\
j^{\prime} k^{\prime} l^{\prime} m^{\prime}}} X_{j k l m 0} X_{j^{\prime} k^{\prime} l^{\prime} m^{\prime} 0} \int x_{0}^{j+j^{\prime}} p_{x 0}^{k+k^{\prime}} y_{0}^{l+l^{\prime}} p_{y 0}^{m+m^{\prime}} \\
& \times \rho_{0} d v_{0}
\end{aligned}
$$

with $j+k+l+m \leq q$ and $j^{\prime}+k^{\prime}+l^{\prime}+m^{\prime} \leq q$. If the contribution from the most relevant order $q,\left\langle x_{f}^{2}\right\rangle_{q}-$ $\left\langle x_{f}^{2}\right\rangle_{q-1}$, is much larger than its corresponding monochromatic contribution, $\left\langle x_{f}^{2}\right\rangle_{q, \Delta_{\delta}=0}-\left\langle x_{f}^{2}\right\rangle_{q-1, \Delta_{\delta}=0}$, the correctors should be placed in dispersive locations possibly together with achromatic correctors to cancel the arising geometric aberrations. In the opposite case, only achromatic correctors should be placed.

\section{OPTIMIZATION OF THE CLIC BEAM DELIVERY SYSTEM}

The CLIC BDS consists of a collimation section and a final focus system (FFS). The Twiss functions of the BDS are plotted in Fig. 1. The collimation section has a length of about $2 \mathrm{~km}$. The first $70 \mathrm{~m}$ serve as a matching section between the main linac and the BDS. After these $70 \mathrm{~m}$ the $\alpha$ functions are zero and therefore this location is taken as the initial location for the computation of the transfer map. The horizontal and vertical normalized beam emittances are $\epsilon_{x}=68 \times 10^{-8} \mathrm{~m}$ and $\epsilon_{y}=1 \times 10^{-8} \mathrm{~m}$, with a relativistic gamma of $\gamma=3 \times 10^{6}$. The full energy width of the beam is $\Delta_{\delta}=0.01$. The rms beam sizes at the IP are computed using Eq. (7) and plotted up to the ninth order in Fig. 2. The nominal beam as well as the monochromatic beam $\left(\Delta_{\delta}=0\right)$ are shown leading to the conclusion that most of the aberrations are chromatic. Only sextupolar and octupolar geometric aberrations appear in the vertical plane. It is striking that the vertical aberrations are relevant up to the highest orders. The most relevant horizontal aberrations are the first order dispersion and the chromaticity (of sextupolar order). The total number of nonzero coefficients of the ninth order transverse map is 4002, of which 2070 are horizontal and 1932 are vertical. These large numbers make the evaluation of the rms beam size very slow. A better approach for an optimization of the

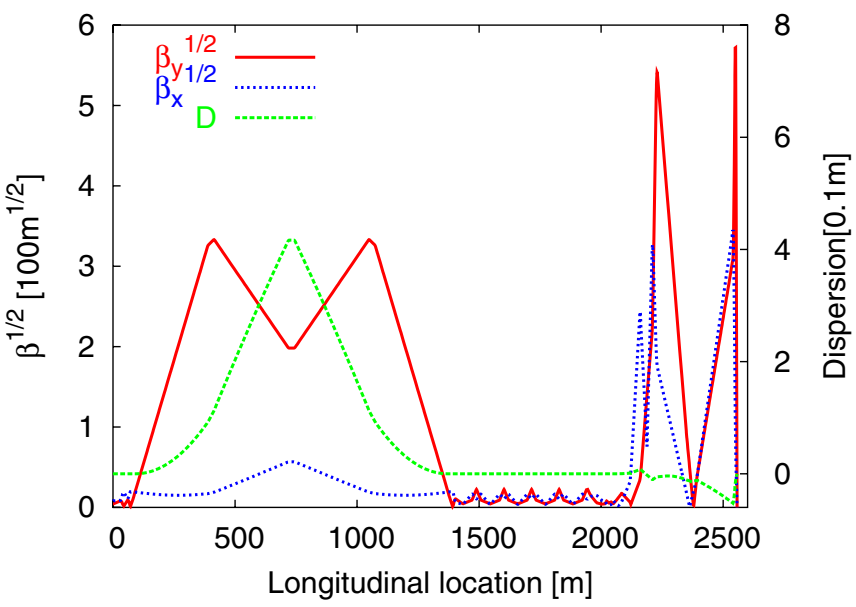

FIG. 1. (Color) Twiss functions of the CLIC beam delivery system. 


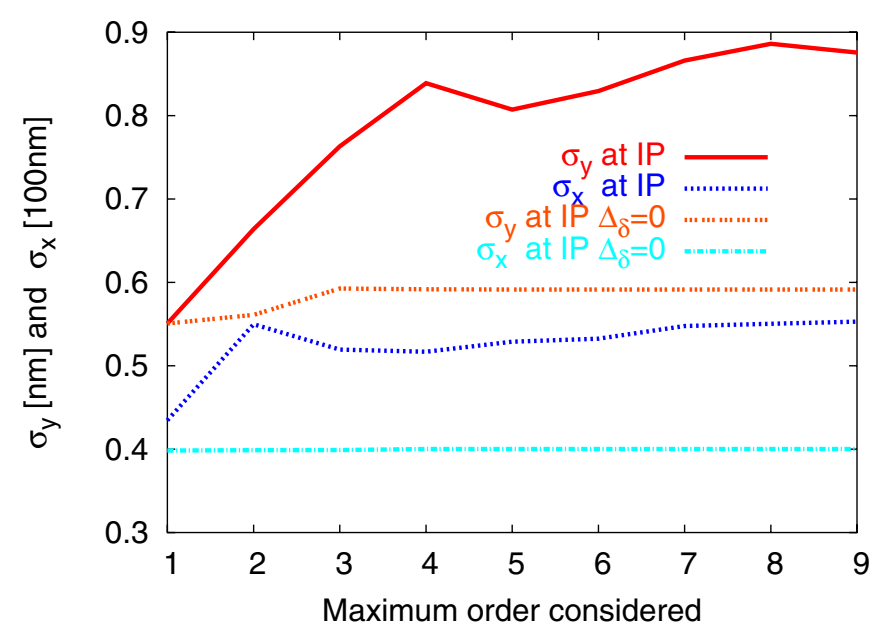

FIG. 2. (Color) Horizontal and vertical rms beam sizes at the CLIC interaction point as a function of the maximum order considered in the transfer map. Both the nominal and monochromatic beams are considered. The horizontal aberrations are purely chromatic and only a small part of the vertical aberrations are geometric.

beam sizes is to consider the collimation section and the final focus separately.

Figure 3 shows the rms beam sizes at the end of the collimation system versus the maximum order considered in the map, again for the nominal and the monochromatic beams. It is obvious that the collimation section needs only a better adjustment of the chromaticity sextupoles. In this context this is efficiently achieved by matching the rms beam sigmas of order 2 to those of order 1 by varying the strengths of the chromaticity sextupoles. In principle, any optimization algorithm can be used to carry out this task. The code MAPCLASS [10] was expressly written for this

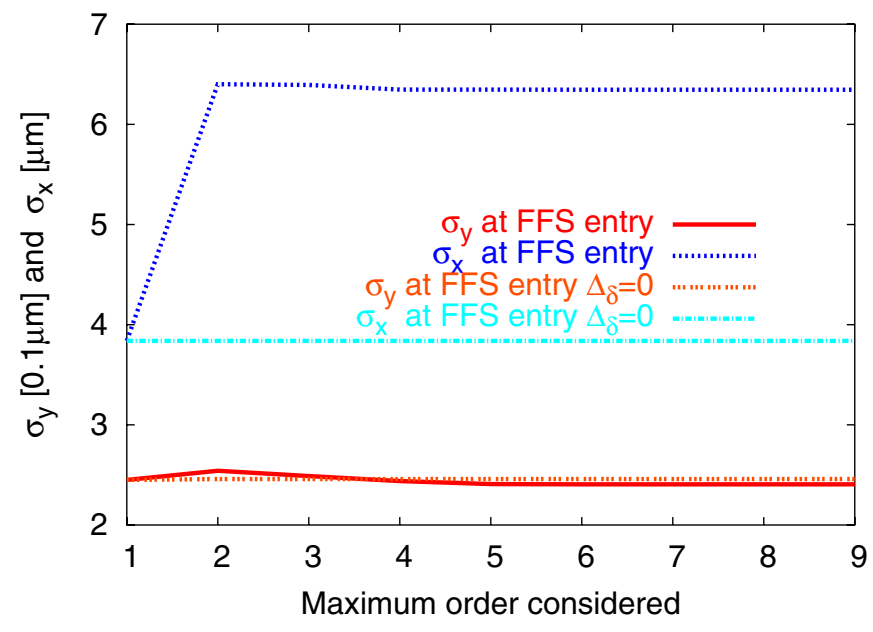

FIG. 3. (Color) Horizontal and vertical rms beam sizes at the end of the CLIC collimation section as a function of the maximum order considered in the transfer map. Both the nominal and monochromatic beams are considered. The horizontal aberrations are purely chromatic.

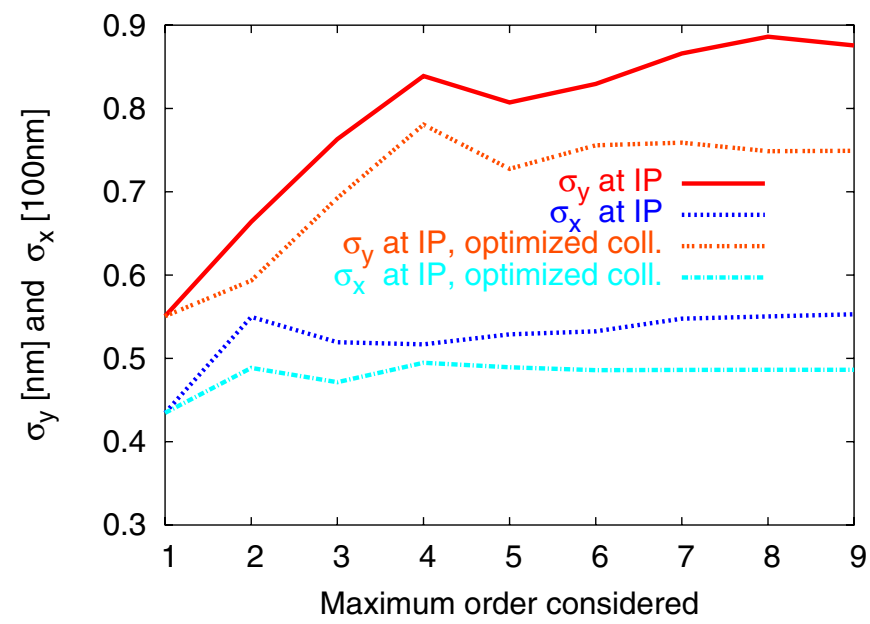

FIG. 4. (Color) Horizontal and vertical rms beam sizes at the CLIC IP for the nominal collimation system and the one with optimized chromaticity.

purpose with an implementation of the simplex method [11]. The resulting beam sizes at the IP are shown in Fig. 4 versus order. The direct effect on the second order is patent. More interestingly, the contributions from orders above six have been significantly reduced both in the horizontal and vertical planes.

The remaining aberrations originate entirely in the FFS. The CLIC FFS has been designed based on the local chromaticity correction scheme proposed in [2]. This scheme basically consists of two pairs of sextupoles, one pair for the horizontal plane and the other for the vertical. The sextupoles of each pair are separated by a $180^{\circ}$ phase advance to compensate chromaticities and the subsequent geometrical aberrations. The compensation of the higher order aberrations can be achieved by correctors of the appropriate order arranged like the sextupoles. We assume that the pairs of sextupoles are combined magnets includ-

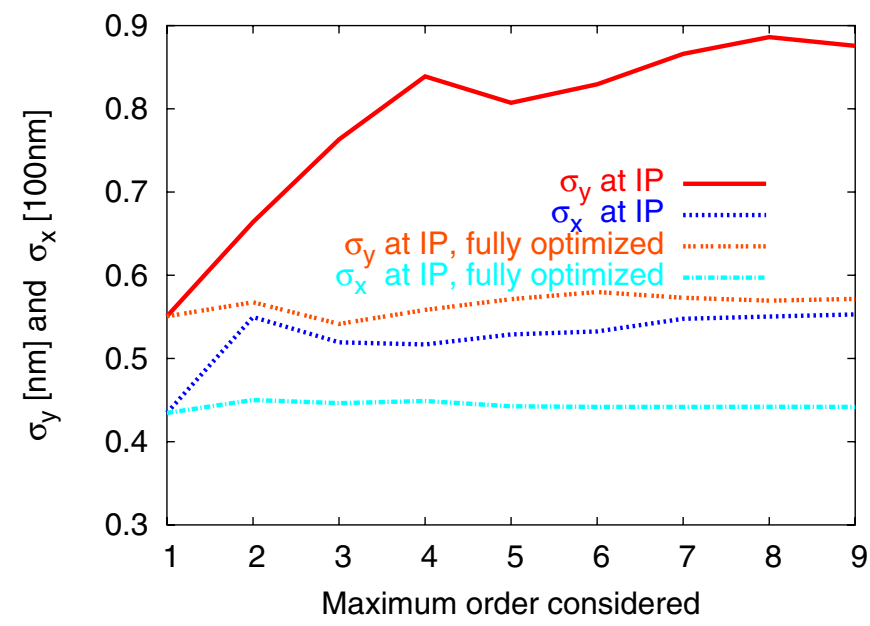

FIG. 5. (Color) Horizontal and vertical rms beam sizes at the CLIC IP for the nominal BDS and the one fully optimized. 


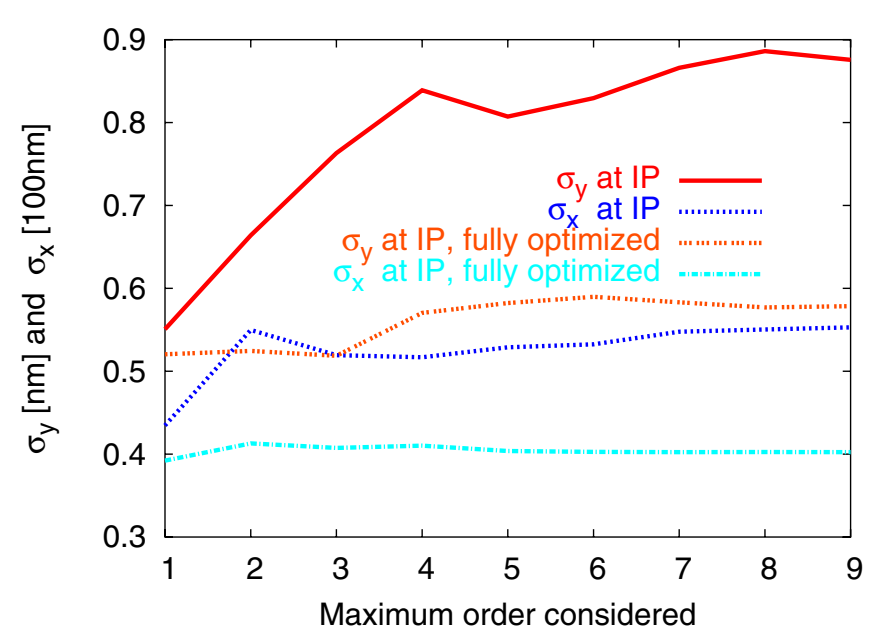

FIG. 6. (Color) Horizontal and vertical rms beam sizes at the CLIC IP for the nominal BDS and the one linearly and nonlinearly optimized.

ing octupolar and decapolar magnetic components. Using all these nonlinear elements (sextupoles, octupoles, and decapoles) in the FFS the rms beam sizes at the IP are minimized with the simplex method. Initially the optimization works efficiently by truncating the map at forth order. After a few iterations it is mandatory to increase the order up to sixth order. Otherwise the rms beam size is minimized at fourth order but increases in the following orders. The required computing time largely increases for this higher order. The result of the optimization of the FFS is shown in Fig. 5 together with the initial configuration. This confirms the compensation of the aberrations up to the higher orders.

Exactly the same algorithm can be used to optimize the linear parameters. Now that the nonlinear aberrations have been compensated it is possible to focus more (decrease the beta functions at the IP) in order to reduce the beam size and gain luminosity. The rms beam sizes up to order 5 are minimized again using the simplex method as before. The difference now is that only the strengths of the quadrupoles are used in the minimization. The result is shown in Fig. 6 together with the initial configuration. Both the horizontal and vertical beta functions have been reduced at the IP as can be seen at the first order of the plot. The horizontal beta function at the IP has been reduced by $19 \%$. The horizontal nonlinear aberrations stay well compensated, while in the vertical plane small aberrations have arisen as a conse- quence of the focusing. This implies that for further reduction of the beam sizes more iterations correcting nonlinear and linear orders are required.

The real benefit of reducing the rms size at the IP is luminosity, and therefore it has been computed for all the former stages of the optimization. Bunches of 10000 electrons have been tracked trough the CLIC BDS using PLACET [12]. The same beam parameters as mentioned above have been used and the effect of synchrotron radiation has been included, which is not taken into account by the described optimization procedure. The luminosity has been computed using the code GUINEA-PIG [13]. The relative reduction of the beam sizes with and without radiation together with the relative luminosity increase is shown in Table I. The total luminosity $\left(L_{\text {tot }}\right)$, the luminosity coming from the collisions with energy larger than $99 \%$ of the maximum energy $\left(L_{1 \%}\right)$, and their ratio $\left(L_{1 \%} / L_{\text {tot }}\right)$ are shown in the table. Both the total luminosity and the luminosity in the energy peak increase as the horizontal rms beam size gets smaller. It is interesting to see that after the first step the variation of the rms sizes with radiation is smaller than the variation of the rms sizes without radiation. Indeed the relative changes of the luminosities seem to be more related to the rms sizes without radiation, reinforcing the described optimization algorithm. The ratio of the luminosities slightly decreases but not in a significant manner.

\section{CONCLUSIONS AND OUTLOOK}

A general algorithm has been developed for the nonlinear optimization of beam lines. This optimization is conceived as a second step after the beam line optics has been designed. It has been applied to the CLIC BDS, obtaining a luminosity increase of $45 \%$ over the nominal design luminosity. The feasibility of the proposed corrector configuration still needs to be assessed from the point of view of magnet design, but also alternative configurations are under study. Further applications of this algorithm can be found in [14-16].

\section{ACKNOWLEDGMENTS}

Thanks to D. Schulte for setting up the PLACET and GUINEA-PIG codes. F. Schmidt provided invaluable help concerning the use of MAD-X and PTC. Many thanks to F. Zimmermann for useful suggestions. Thanks also to

TABLE I. CLIC luminosities for the different optimizations stages. All numbers are in percent units.

\begin{tabular}{|c|c|c|c|c|c|c|c|}
\hline Case & $-\frac{\Delta \sigma_{x}}{\sigma_{x}^{\operatorname{mss}}}($ no rad $)$ & $-\frac{\Delta \sigma_{x}}{\sigma_{x}^{\text {rss }}}(\mathrm{rad})$ & $-\frac{\Delta \sigma_{y}}{\sigma_{y}^{\text {rms }}}($ no rad $)$ & $-\frac{\Delta \sigma_{y}}{\sigma_{y}^{\text {rms }}}(\mathrm{rad})$ & $\frac{\Delta L_{\text {tot }}}{L_{\text {tot }}}$ & $\frac{\Delta L_{1 \%}}{L_{1 \%}}$ & $\frac{L_{1 \%}}{L_{\mathrm{tot}}}$ \\
\hline Nominal & 0 & 0 & 0 & 0 & 0 & 0 & 43 \\
\hline Corrected collimation section & 12 & 30 & 14 & 58 & 9 & 6 & 42 \\
\hline Corrected FFS nonlinearities & 20 & 35 & 35 & 69 & 31 & 19 & 39 \\
\hline Lower $\beta_{x}$ and $\beta_{y}$ at IP & 27 & 37 & 34 & 64 & 45 & 29 & 38 \\
\hline
\end{tabular}


A. Faus-Golfe, J. Resta, D. Schulte, J. Payet, F. Ruggiero, and F. Zimmermann for their motivation and useful discussions.

[1] David C. Carey, in Proceedings of the Particle Accelerator Conference, San Francisco, CA, 1973 (IEEE, Piscataway, NJ, 1973), p. 493.

[2] P. Raimondi and A. Seryi, Phys. Rev. Lett. 86, 3779 (2001).

[3] F. Zimmermann, R. Helm, and J. Irwin, in Proceedings of the Particle Accelerator Conference, Dallas, TX, 1995 (IEEE, Piscataway, NJ, 1995), p. 710.

[4] F. Zimmermann, Nucl. Instrum. Methods Phys. Res., Sect. A 364, 231 (1995).

[5] O. Napoly and J. Payet, in Proceedings of the Nanobeam Workshop, Lausanne, Switzerland, 2002 (CERN, Geneva, 2002).

[6] A. Faus-Golfe, in Proceedings of the Nanobeam Workshop, Lausanne, Switzerland, 2002 (Ref. [5]).

[7] F. Zimmermann, H. Burkhardt, T. Risselada, F. Schmidt, and H.J. Schreiber, in Proceedings of the 18th International Conference on High Energy Accelerators (HEACC2001), Tsukuba, Japan, 2001 (CERN Report
No. CERN-SL-2001-010 AP, 2001).

[8] H. Grote and F. Schmidt, Report No. CERN-AB-2003024, ABP.

[9] E. Forest, F. Schmidt, and E. McIntosh, KEK Report No. 2002-3.

[10] R. Tomás, CERN Report No. CERN-AB-Note-017 ABP, 2006.

[11] J. A. Nelder and R. Mead, Computer Journal (UK) 7, 308 (1965).

[12] D. Schulte, T. E. D'Amico, G. Guignard, and N. Leros, in Proceedings of the Particle Accelerator Conference, Chicago, IL, 2001 [CERN Report No. CERN/PS 2001 028 (AE), 2001].

[13] D. Schulte, in Proceedings of the International Computational Accelerator Physics Conference (ICAP98), Monterey, CA, 1998, eConf C980914, R580 (1998).

[14] R. Tomás, CERN Report No. CERN-OPEN-2006-023, 2006.

[15] A. Faus-Golfe, F. Zimmermann, and J. Resta-Lapez, in Proceedings of the 10th European Particle Accelerator Conference, Edinburgh, UK, 2006 (IOP, London, 2006).

[16] P. R. Jarnhus and R. Tomás, "Optimization of the CLIC Final Focus Dispersion without Using Extra Multipolar Components," CERN AB Note (to be published). 